\title{
Risk Factors for Colonisation or Infection with Carbapenem Resistant Escherichia Coli and Klebsiella Pneumoniae
}

\author{
Volkan Atmis *, [MD] \\ Mesut Gumussoy ${ }^{1}$, [MD] \\ Murat Cinel $^{2}$, [MD] \\ Sule Canlar ${ }^{3}$, [MD] \\ Nadire Yesim Cetinkaya Sardan ${ }^{4}$, [MD] \\ 1, Mesut Gumussoy, Tokat Niksar Public Hospital, \\ Internal Medicine, Specialist, \\ 2, Murat Cinel, Ankara University, Ibni Sina Hospital, \\ Endocrinology and Internal Medicine, Specialist, \\ 3, Sule Canlar, Aksaray Public Hospital, Endocrinology \\ and Internal Medicine, Specialist, \\ 4, Nadire Yesim Cetinkaya Sardan, Guven Hospital, \\ Infectious diseases and Internal Medicine, Professor \\ "Corresponding Author: Volkan Atmis, \\ Bursa Yuksek Ihtısas Education and Research Hospital, \\ Geriatrics and Internal Medicine, Specialist, \\ Bursa, TURKEY \\ DOI: https://doi.org/10.32552/2018.ActaMedica.306 \\ e-mail:volkanatmis@hotmail.com
}

\section{wa ABSTRACT Cem}

Objective: Since 1991, carbapenem resistance is being reported in an increasing fashion worldwide. It is expected to increase due to increasing frequency of international travels. Carbapenem resistant Escherichia coli and Klebsiella pneumoniae can cause colonization or clinical infection. Treatment of carbapenem resistant Escherichia coli or Klebsiella pneumoniae is not precise and we have limited choices of antibiotics. This brings increasing morbidity and mortality with itself. We aimed to find out risk factors for colonization or infection with carbapenem resistant Escherichia coli and Klebsiella pneumoniae in this study

Materials and Methods: With the decision of Adult Hospital Infection Control Comittee, a surveillance has been started among adult patients ( $>18$ years of age) at Hacettepe University Hospitals at June 2009. A perianal swab culture has been obtained weekly by Adult Hospital Infection Control nurses from patients included in surveillance program. In this study, carried on adult patients hospitalized at Hacettepe University hospitals between 01.06.2009-31.12.2010, risk factors for colonization or infection with carbapenem resistant Escherichia coli and Klebsiella pneumoniae, those are detected via surveillance cultures or clinical specimens, were investigated.

Results: According to results of the multivariate analysis of the study; being hospitalized in an internal ward (any ward other than a surgical ward) $(\mathrm{p}=0,005)$, Diabetes Mellitus ( $\mathrm{p}=0,010)$, immunosuppression $(\mathrm{p}<0,001)$, hospitalization history $(\mathrm{p}<0,005)$, burn history $(\mathrm{p}=0,026)$, central venous line history $(\mathrm{p}=0,012)$, arterial line history $(\mathrm{p}=0,010)$, feeding or nasogastric tube history $(\mathrm{p}<0,001)$, sulbactam/ampicillin use $(\mathrm{p}<0,001)$, glycopeptide use $(\mathrm{p}=0,003)$ and carbapenem or piperacillin/tazobactam use $(\mathrm{p}<0,001)$ were all detected to be risk factors.

Key Words: Carbapenem resistant E.coli and K.pneumonia, colonization or infection, risk factors

Received: 06 September 2018, Accepted: 18 September

2018, Published online: 30 September 2018

\section{INTRODUCTION}

Gram-negative bacteria are still among leading factors in both community-based and health-care-associated infections. Various effective and safe antibiotics are in use since the Second World War. However, the widespread and inappropriate use of these drugs has led to the growing problem of antimicrobial resistance. Today, multiple antibiotic resistance in gram-negative bacilli, either community-based or health-care-related infectious agents, have reached the dimensions that threaten human health [1]. Carbapenem group antibiotics have long been the strongest weapon of clinicians for the treatment of healthcare-associated infections that are caused by resistant gram-negative bacilli. Unfortunately, carbapenem resistance, which was extremely rare in the last few years, is increasing worldwide among the Enterobacteriaceae family, especially Klebsiella pneumoniae. The emergence and spread of non-carbapenem sensitive (moderately resistant or resistant) Escherichia coli has also become an important clinical problem in recent years [2-7].

Carbapenem-resistant E. coli and K. pneumoniae strains have been reported to cause clinical infections such as bloodstream infections, ventilator-associated pneumonia and urinary system infections, 
or asymptomatic colonization in tertiary hospitals and state hospitals [8-16]. Some of these infections are sporadic health care-related infections and some are clonal outbreaks. There are limited studies of the efficacy of avibactam in combination with ceftazidime or aztreonam [17]. Antibiotic options that can be used to treat infections caused by resistant strains of E. coli and K. pneumoniae are extremely limited and there is no hope that this problem will be solved in the near future. For this reason, while trying to slow the development of resistance by using antibiotics on the right indictment, on the one hand, it is very important to prevent the spread of these strains in the hospital and between hospitals.

Although carbapenem resistance has been reported in E. coli and K. pneumoniae strains at many centers in our country, epidemiological studies to identify risk factors for colonization / infections of these microorganisms are limited. In March 2009, recommendations for controlling carbapenem resistant Enterobacteriaceae were published by Centers for Disease Control and Prevention in hospitals[18]. Taking these suggestions into account, an active surveillance program has been initiated to detect infected or colonized patients with carbapenem resistant $\mathrm{E}$. coli or K. pneumoniae in Hacettepe University Adult and Oncology Hospitals. In this study, it was aimed to determine the risk factors for carbapenem resistant E. coli and K. pneumoniae infection/colonization in adult patients in Hacettepe University Hospitals by comparing the cases covered by the above mentioned surveillance program with the appropriate control patients.

\section{MATERIALS AND METHODS}

Surveillance for carbapenem resistant E. coli and K. pneumoniae started in June 2009 on adult patients hospitalized at Hacettepe University Hospitals (> 18 years) under the decision of the Adult Hospital Infection Control Committee. All patients in the Adult Hospital Intensive Care Units (ICU) (Internal Medicine, General Surgery, Brain Surgery, Neurology, Burn, Anesthesia and ICU after Anesthesia), neutropenic patients in the Internal Diseases Services, bone marrow and solid organ transplant patients were included in this surveillance. Perianal swab cultures were taken once a week from the patients who were included in Surveillance. If carbapenem resistant $\mathrm{E}$. coli and $\mathrm{K}$. pneumoniae were isolated from surveillance cultures from a service / ICU, perianal culture was continued to be taken for once in two weeks. If no carbapenem resistant $\mathrm{E}$. coli and $\mathrm{K}$. pneumoniae were isolated for four weeks in cultures taken from a service / ICU for four weeks, surveillance was conducted by perianal culture once a month. Microbiological studies related to surveillance cultures have been carried out in the Research Laboratory of Infectious Diseases Unit of Internal Medicine Department.

Adult patients ( $>18$ years of age) with carbapenem resistant E. coli or K. pneumoniae in at least one perianal swab culture or one of their clinical specimens, hospitalized for $>72$ hours between 01.06.2009 and 31.12.2010 in the units covered by the surveillance, were accepted as case. The control group was randomly selected from adult patients, hospitalized for $>72$ hours in the same time interval as cases in the same wards with no carbapenem resistant E. coli or K. pneumoniae in perianal swab specimens and clinical specimens, Two control patients were identified for each case.

Perianal specimens obtained from the patients with sterile ointment were sent to the Investigative Laboratory of Infectious Diseases Unit for Infectious Diseases, Department of Internal Medicine, in a tube containing $5 \mathrm{ml}$ of tryptic strain broth (Oxoid, UK) containing $10 \mu \mathrm{g}$ ertapenem disc (Oxoid, UK). After incubation for $6-7$ hours at $37^{\circ} \mathrm{C}$ in this liquid medium specimens was passed to the Mac Conkey agar (Oxoid, UK) and these mediums were incubated overnight at $37^{\circ} \mathrm{C}$. The following day, typical colonies of Escherichia coli or Klebsiella pneumoniae were identified by API 20E (BioMerieux, France), carbapenem sensitivity was determined by imipenem Etest (AB Biodisk, UK). Strains with an MIC value of $>$ $8 \mu \mathrm{g} / \mathrm{ml}$ were defined as carbapenem-resistant $\mathrm{K}$. pneumoniae or E. coli.

The files of the cases and the patients in the control group were reviewed and demographic information and possible risk factors were recorded in a standard form. Data were recorded on the entire length of stay for control patients, and were recorded until the first date of detection of carbapenem resistant E. coli or K. pneumoniae for the cases.

Analysis of the data was performed in SPSS for Windows 11.5 package program. Descriptive statistics are shown as mean \pm standard deviation for continuous variables or as median (minimum-maximum), nominal variables as number of cases and (\%). The significance of differences between the groups in terms of means was determined by Student's $t$ test, and medians was examined by Mann Whitney $U$ test. Nominal variables were assessed by Pearson's Chi-Square or Fisher's Exact Result Chi-Square test. Variables that differ significantly between case and control groups as a result of univariate statistical analyzes were included in the Multivariate Logistic Regression model. Backward stepwise screening 
method was used to identify the factors that have the most determinants without discriminating between control and case groups. The odds ratio, 95\% confidence interval, wald statistics and significance levels for each variable were calculated. For $p<0.05$, the results were considered statistically significant

\section{RESULTS}

Between 01.06.2009 and 31.12.2010, a total of 43,312 patients were reviewed, 38,372 of which were in Adult Hospital and 5,040 in Oncology Hospital. During this period, a total of 137 patients (102 patients with carbapenem resistant K. pneumoniae and 38 patients with carbapenem resistant E. coli) who met the above-mentioned case definition were detected. Seven of these patients were excluded from the study because their file records were not available. K. pneumoniae / E.coli was detected in 130 cases ( 93 carbapenem resistant K. pneumoniae, 37 carbapenem resistant E. coli) in perianal culture and 12 clinical specimens in file records. E. coli was detected in one patient's blood culture, in one patient's blood and catheter culture, K. pneumonia was detected inn four patients' urine culture, in one patient's pus culture and deep tracheal aspiration (DTA) in two patients, in two patients' pus and perianal culture, in blood and perianal culture in one patient. In 83 of patients K.pneumonia was detected only in perianal culture and E.coli only in perianal cultures 35 patients (Table 1). The distribution of the cases according to the wards is presented in Table 2.

Table 1. Distribution of carbapenem resistant K. pneumoniae / E.coli cultures in patients involved in the study by sample types

\begin{tabular}{|l|c|c|c|}
\hline Isolates & Number $(\%)$ & K.pneumoniae & E.coli \\
\hline & & Number $(\%)$ & $35(\% 26)$ \\
\hline Perianal culture & $118(\% 90)$ & $83(\% 63$ & $1(\% 0,76)$ \\
\hline Blood culture & $1(\% 0,76)$ & $0(\% 0)$ & $1(\% 0,76)$ \\
\hline Blood +catheter culture & $1(\% 0,76)$ & $0(\% 0)$ & $0(\% 0)$ \\
\hline Urine culture & $4(\% 3)$ & $4(\% 3)$ & $0(\% 0)$ \\
\hline Pus culture & $1(\% 0,76)$ & $1(\% 0,76)$ & $0(\% 0)$ \\
\hline DTA culture & $2(\% 1,5)$ & $2(\% 1,5)$ & $0(\% 0)$ \\
\hline Pus + perianal culture & $2(\% 1,5)$ & $2(\% 1,5)$ & $0(\% 0)$ \\
\hline Blood + perianal culture & $1(\% 0,76)$ & $1(\% 0,76)$ & $37(\% 29)$ \\
\hline Total & $130(\% 100)$ & $93(\% 71)$ & \\
\hline
\end{tabular}

DTA: deep tracheal aspiration

Table 2. Isolated microorganisms by wards

\begin{tabular}{|l|c|c|c|}
\hline Ward & E.coli & K.pneumoniae & Total \\
\hline 76 (Internal Medicine) & 0 & 14 & 2 \\
\hline 76 (Infectious diseases) & 1 & 1 & 2 \\
\hline 75 (Urology) & 2 & 0 & 35 \\
\hline 74 (NICU) & 5 & 30 & 3 \\
\hline 64 (THBS-transplant) & 2 & 1 & 7 \\
\hline 63 (Burn Unit) & 1 & 6 & 5 \\
\hline AICU & 1 & 4 & 19 \\
\hline GSICU & 7 & 12 & 9 \\
\hline IMICU & 2 & 7 & 9 \\
\hline NSICU & 2 & 7 & 2 \\
\hline
\end{tabular}

DTA: deep tracheal aspiration 


\begin{tabular}{|l|c|c|c|}
\hline 95 & 3 & 5 & 8 \\
\hline 94 & 5 & 3 & 8 \\
\hline 93 & 2 & 1 & 3 \\
\hline 91 & 4 & 2 & 6 \\
\hline Total & 37 & 93 & 130 \\
\hline
\end{tabular}

NICU: Neurology ICU, THVS: Thorax heart and vessel surgery, GSICU: General surgery ICU, IMICU: Internal medicine ICU, NSICU: Neurosurgery ICU

A total of 404 patients; 130 cases and 274 controls, were included in the study. Variables with statistically significant risk factors in univariate analysis were; the mean age of the patients, hospital stay in internal wards, DM, CAD-CHF or HT combined, CKD, CLD, $\mathrm{CLiD}$, Cancer history, immunosuppression (transplantation, neutropenia, chemotherapy, HIV or steroid use), transplantation, neutropenia, chemotherapy, steroid use, previous hospitalization, ICU admission, nursing home stay, burn history, central catheterization, arterial catheterization, urinary catheterization history, proton pump inhibitor (PPI) use, feeding/nasogastric tube insertion history, gastrostomy, enterostomi, colostomy, tracheotomy history, diarrhea, total parenteral nutrition (TPN), elective surgery history (protective), acute organ damage, sulbactam ampicillin (SAM), cefazolin, cefepime, ciprofloxacin, levofloxacin, moxifloxacin, amikacin, gentamicin, netilmisin, clarithromycin, piperacillin/ tazobactam, sulperazon, metronidazole, rifampicin, colistin, vancomycin, teicoplanin, linezolid, tigecycline, carbapenem, fluconazole, voriconazole, amphotericin B (Amph B), caspofungin use each. Sex, duration of hospital stay, trauma history, noninvasive mechanical ventilation, invasive mechanical ventilation, $\mathrm{H} 2$ blocker use, sucralfate use, drainage catheter use, rheumatological disease, urgent surgical intervention, cefuroxime, ceftazidime, ceftriaxone, erythromycin, TMP-SMX, İtraconazole use each were the variables statistically non-significant in univariate analysis. Univariate analysis results are shown Tables3-6; Demographics and wards of the patients in Table 3, comorbid diseases and medical histories in Table 4, invasive intervention histories in Table 5 and drug and antibiotic use histories in Table 6.

Table 3. Demographics and wards

\begin{tabular}{|l|c|c|c|}
\hline Variables & Number $(\%)$ & K.pneumoniae & E.coli \\
\hline Age & $59(0-92)$ & $49(1-101)$ & $<0,001$ \\
\hline Sex & & & - \\
\hline Male & $55(\% 42,3)$ & $140(\% 51,1)$ & 0,099 \\
\hline Female & $75(\% 57,7)$ & $134(\% 48,9)$ & - \\
\hline Ward & & & $<0,001$ \\
\hline Surgery & $48(\% 36,9)$ & $216(\% 78,8)$ & $58(\% 21,2)$ \\
\hline Internal & $82(\% 63,1)$ & 58 & $<$ \\
\hline
\end{tabular}

Table 4. Comorbid diseases and medical history

\begin{tabular}{|l|c|c|c|}
\hline Variables & & & P value \\
\hline & Case Group & Control Group & $<0,001$ \\
\hline Diabetes mellitus & $39(\% 30,0)$ & $31(\% 11,3)$ & $<0,001$ \\
\hline CAD, CHF,HT & $65(\% 50,0)$ & $84(\% 30,7)$ & $<0,001$ \\
\hline Chronic kidney disease & $21(\% 16,2)$ & $13(\% 4,7)$ & 0,017 \\
\hline Chronic lung disease & $13(\% 10,0)$ & $11(\% 4,0)$ & 0,010 \\
\hline Chronic liver disease & $4(\% 3,1)$ & $0(\% 0)$ & $<0,001$ \\
\hline Cancer & $46(\% 35,4)$ & $51(\% 18,6)$ & $<0,001$ \\
\hline Immunosuppression & $72(\% 55,4)$ & $37(\% 13,5)$ & \\
\hline
\end{tabular}




\begin{tabular}{|l|c|c|c|}
\hline Transplantation & $9(\% 6,9)$ & $2(\% 0,7)$ & $<0,001$ \\
\hline Neutropenia & $11(\% 8,5)$ & $3(\% 1,1)$ & $<0,001$ \\
\hline Hospital stay history & $52(\% 40,0)$ & $28(\% 10,2)$ & 0,723 \\
\hline Duration of Hospital stay & $0(0-4)$ & $1(0-38)$ & $<0,001$ \\
\hline ICU stay history & $8(\% 6,2)$ & $0(\% 0)$ & 0,033 \\
\hline Nursing home stay history & $3(\% 2,3)$ & $1(\% 0,4)$ & 0,015 \\
\hline Burn history & $5(\% 3,8)$ & $0(\% 0)$ & 0,103 \\
\hline Trauma history & $2(\% 1,5)$ & $3(\% 1,1)$ & 0,392 \\
\hline Rheumatological disease & $3(\% 2,3)$ & $3(\% 1,1)$ & 0,015 \\
\hline Diarrhea & $7(\% 5,4)$ & $18(\% 6,6)$ & $<0,001$ \\
\hline Acute organ damage & $46(\% 35,4)$ & $1 \%$ & \\
\hline
\end{tabular}

Table 5. Invasive intervention history

\begin{tabular}{|c|c|c|c|}
\hline Variables & Case Group & Control Group & $P$ value \\
\hline $\begin{array}{l}\text { Central catheterization } \\
\text { history }\end{array}$ & $80(\% 61,5)$ & $17(\% 6,2)$ & $<0,001$ \\
\hline $\begin{array}{l}\text { Arterial catheterization } \\
\text { history }\end{array}$ & $38(\% 29,2)$ & $7(\% 2,6)$ & $<0,001$ \\
\hline $\begin{array}{l}\text { Urinary catheterization } \\
\text { history }\end{array}$ & $70(\% 53,8)$ & $58(\% 21,2)$ & $<0,001$ \\
\hline $\begin{array}{l}\text { Noninvasive mechanical } \\
\text { ventilation }\end{array}$ & $2(\% 1,5)$ & $1(\% 0,4)$ & 0,243 \\
\hline $\begin{array}{l}\text { Invasive } \quad \text { mechanical } \\
\text { ventilation }\end{array}$ & $71(\% 54,6)$ & $166(\% 60,8)$ & 0,255 \\
\hline $\begin{array}{l}\text { Feeding /nasogastric tube } \\
\text { use }\end{array}$ & $71(\% 54,6)$ & $14(\% 5,1)$ & $<0,001$ \\
\hline Gastrostomy use & $21(\% 16,2)$ & $1(\% 0,4)$ & $<0,001$ \\
\hline Enterostomy use & $5(\% 3,8)$ & $0(\% 0)$ & 0,003 \\
\hline Colostomy use & $5(\% 3,8)$ & $2(\% 0,7)$ & 0,038 \\
\hline Tracheotomy use & $22(\% 16,9)$ & $2(\% 0,7)$ & $<0,001$ \\
\hline Drainage catheter use & $15(\% 11,5)$ & $42(\% 15,3)$ & 0,307 \\
\hline Total parenteral nutrition & $28(\% 21,5)$ & $2(\% 0,7)$ & $<0,001$ \\
\hline \multicolumn{4}{|l|}{ Surgical intervention } \\
\hline Absent & $74(\% 56,9)$ & $93(\% 33,9)$ & - \\
\hline Urgent & $11(\% 8,5)$ & $10(\% 3,6)$ & 0,485 \\
\hline Elective & $45(\% 34,6)$ & $171(\% 62,4)$ & $<0,001$ \\
\hline
\end{tabular}

Table 6. Drug-antibiotic use history

\begin{tabular}{|l|c|c|c|}
\hline Variables & Case Group & Control Group & P value \\
\hline Steroid & $47(\% 36,2)$ & $26(\% 9,5)$ & $<0,001$ \\
\hline Chemotherapy & $17(\% 13,1)$ & $11(\% 4,0)$ & $<0,001$ \\
\hline PPI use & $105(\% 80,8)$ & $95(\% 34,7)$ & 0,001 \\
\hline H2 blocker use & $49(\% 37,7)$ & $87(\% 31,8)$ & 0,238 \\
\hline Sucralfate use & $3(\% 2,3)$ & $2(\% 0,7)$ & $<0,001$ \\
\hline Sulbactam ampicillin & $56(\% 43,1)$ & $52(\% 19,0)$ & \\
\hline
\end{tabular}




\begin{tabular}{|c|c|c|c|}
\hline Cefazolin & $2(\% 1,5)$ & $49(\% 17,9)$ & $<0,001$ \\
\hline Cefuroxime & $1(\% 0,8)$ & $0(\% 0,0)$ & 0,322 \\
\hline Ceftazidime & $1(\% 0,8)$ & $1(\% 0,4)$ & 0,541 \\
\hline Ceftriaxone & $4(\% 3,1)$ & $6(\% 2,2)$ & 0,733 \\
\hline Cefepime & $5(\% 3,8)$ & $0(\% 0,0)$ & 0,003 \\
\hline Ciprofloxacin & $20(\% 15,4)$ & $6(\% 2,2)$ & $<0,001$ \\
\hline Levofloxacin & $9(\% 6,9)$ & $4(\% 1,5)$ & 0,006 \\
\hline Moxifloxacin & $6(\% 4,6)$ & $3(\% 1,1)$ & 0,034 \\
\hline Amikacin & $8(\% 6,2)$ & $4(\% 1,5)$ & 0,022 \\
\hline Gentamicin & $4(\% 3,1)$ & $0(\% 0)$ & 0,010 \\
\hline Netilmicin & $4(\% 3,1)$ & $0(\% 0)$ & 0,010 \\
\hline Clarithromycin & $11(\% 8,5)$ & $0(\% 0)$ & $<0,001$ \\
\hline Erythromycin & $1(\% 0,8)$ & $0(\% 0)$ & 0,322 \\
\hline Piperacillin/tazobactam & $54(\% 41,5)$ & $6(\% 2,2)$ & $<0,001$ \\
\hline Sulperazon & $34(\% 26,2)$ & $0(\% 0)$ & $<0,001$ \\
\hline Metronidazole & $14(\% 10,8)$ & $0(\% 0)$ & $<0,001$ \\
\hline Rifampin & $6(\% 4,6)$ & $0(\% 0)$ & $<0,001$ \\
\hline Colistin & $14(\% 10,8)$ & $0(\% 0)$ & $<0,001$ \\
\hline TMP-SMX & $5(\% 3,8)$ & $3(\% 1,1)$ & 0,118 \\
\hline Vancomycin & $56(\% 43,1)$ & $3(\% 1,1)$ & $<0,001$ \\
\hline Teicoplanin & $12(\% 9,2)$ & $2(\% 0,7)$ & $<0,001$ \\
\hline Linezolid & $5(\% 3,8)$ & $0(\% 0)$ & 0,003 \\
\hline Tigecycline & $4(\% 3,1)$ & $1(\% 0,4)$ & 0,039 \\
\hline Carbapenem & $66(\% 50,8)$ & $6(\% 2,2)$ & $<0,001$ \\
\hline Fluconazole & $30(23,1)$ & $0(\% 0)$ & $<0,001$ \\
\hline Voriconazole & $5(\% 3,8)$ & $0(\% 0)$ & 0,003 \\
\hline İtraconazole & $1(\% 0,8)$ & $0(\% 0)$ & 0,322 \\
\hline Amphotericin B & $4(\% 3,1)$ & $0(\% 0)$ & 0,010 \\
\hline Caspofungin & $7(\% 5,4)$ & $0(\% 0)$ & $<0,001$ \\
\hline
\end{tabular}

Statistically significant variables and risk factors in multivariate analysis were; internal ward stay history, DM history, immunosuppression history, burn history, central catheterization, arterial catheterization, nutrition tube or nasogastric tube history, elective surgical history (protective), sulbactam/ampicillin use history, gly copeptide use history, carbapenem or piperacillin/tazobactam use history (Table 7).

Table 7. The factors determined to be the most to determinative to discriminate control and case groups as a Result of Backward Stepwise Logistic Regression Analysis

\begin{tabular}{|l|c|c|c|c|c|}
\hline Variables & Odds ratio & Wald value & P value & \multicolumn{2}{|c|}{$95 \%$ Confidence Interval } \\
\hline & & & & \multicolumn{1}{|c|}{ Lower limit } & Upper limit \\
\hline Internal ward stay & 3,385 & 6,254 & 0,012 & 1,302 & 8,803 \\
\hline DM & 3,613 & 6,031 & 0,014 & 1,296 & 10,073 \\
\hline Immunosuppression & 4,912 & 12,356 & $<0,001$ & 2,022 & 11,930 \\
\hline Burn history & 85,072 & 7,331 & 0,007 & 3,411 & 2121,961 \\
\hline Feeding tube or nasogastric & & & & & 26,663 \\
\hline tube history & 8,434 & 13,183 & $<0,001$ & 2,668 & \\
\hline
\end{tabular}


Carbapenem resistant E.coli and K.pneumonio

\begin{tabular}{|l|c|c|c|c|c|}
\hline $\begin{array}{l}\text { Elective surgical } \\
\text { intervention }\end{array}$ & 0,233 & 6,859 & 0,009 & 0,078 & 0,693 \\
\hline Central catheter history & 3,630 & 6,443 & 0,011 & 1,341 & 9,824 \\
\hline Arterial catheter history & 7,121 & 5,699 & 0,017 & 1,421 & 35,683 \\
\hline Sulbactam/ampicillin use & 6,134 & 14,019 & $<0,001$ & 2,373 & 15,853 \\
\hline Glycopeptide use & 10,756 & 8,577 & 0,003 & 2,194 & 52,730 \\
\hline Piperacillin/tazobactam or & & & & & \\
\hline carbapenem use & 9,699 & 18,424 & $<0,001$ & 3,437 & 27,372 \\
\hline
\end{tabular}

\section{DISCUSSION}

In studies for determining infection risk with carbapenem resistant bacteria, results are often parallel to each other, with differences often appearing due to the lack of evaluation of the relevant risk factor, to the insufficiency of the number cases or to geographical differences[2, 5, 18-20]. Independent risk factors detected in our study as a result of multivariate analysis were; internal ward stay history $(p=0,012), D M(p=0,014)$, immunosuppression $(p<0,001)$, burn trauma $(p=0,007)$, feeding tube or nasogastric tube insertion $(p<0.001)$, surgical intervention $(p=0,009)$, central or arterial catheterization $(p=0,011, p=0,017$ respectively), glycopeptide antibiotic $(p=0.003)$ and piperacillin/tazobactam or carbapenem use $(p<0.001)$. In our study, the presence of CAD, CHF or HT $(p<0,001), C K D(p<0,001)$, CHF $(p<0,001)$ and Chronic lung disease $(p<0,001)$ were statistically significant in the univariate analyzes but not in multivariate analysis, this may be due to primarily to insufficiency of case numbers and this may be secondary to the fact that these diseases often coexist in the same patient. Although the use of PPI was statistically significant in univariate analysis $(p<0.001)$, but was not detected as a risk factor in multivariate analysis. PPI is often used for the gastric protection in patients with multiple co-morbidities, in patients with underlying medical conditions or in patients hospitalized in ICU. PPI use in the control group was $34,7 \%$ and $\mathrm{H} 2$ blocker use was $31,8 \%$, but in the case group these percentages were 80,8 $\%$ and $37,7 \%$ respectively. This increases the likelihood of presence of comorbid diseases increasing the necessity of PPI use. Besides, the use of gastrostomy, enterostomy, colostomy and TPN were statistically significant risk factors in the univariate analysis but not in the multivariate analysis. This seems to be due to the inadequate number of cases primarily. Hospitalization in a ward other than a surgical ward was found to be a risk factor in multivariate analysis $(p=0.005)$. Although this suggests the possibility of the colonization of internal wards, in a study by Borgmann no colonization was detected when environmental factors were scanned for reservoirs, and in a study by Crespo, colonization was detected in stethoscopes and sinks, despite the absence of hand colonization, and the pandemic was resolved after disinfection of the stethoscopes and sinks. There are also other studies showing that source control prevents recurrences [21]. It is known that in our hospital the number of nurses per patient and the number of beds are parallel to each other in internal and surgical wards, and the number of doctors is more in internal wards. This reduces the likelihood of a significant increase in colonization in separate floors and buildings in the internal wards. In addition, a homogenous distribution should be expected in the entire hospital if there is a stethoscope colonization since the antibiotic initiation is in the control infectious diseases unit according to the hospital protocol, and initiation is performed after the examination of the patient by the doctor from infectious diseases unit. From the analysis of subgroups, it was found that among all parameters that were statistically significant as a risk factor in multivariate analysis, only burns were more common in surgical wards (burn wards a subunit of general surgery department) and all other risk factors are more common in patients hospitalized in internal wards. In this case, the presence of the disease, which necessitates the internal ward hospitalization, seems to be a risk factor rather than being hospitalized in an internal ward.

The presence of DM was found to be a significant risk factor in multivariate analysis $(p=0.010)$. This is due to disorders of cellular immunity and phagocyte functions secondary to hyperglycemia, decreased vascularization also contributes to these. Pneumonia, urinary tract infections, skin and soft tissue infections are more common in diabetics. It seems likely that the presence of DM will emerge as a risk factor.

$\mathrm{DM}, \mathrm{CKD}$, cancer, transplantation, neutropenia, chemotherapy use and neutropenia were all found to be statistically significant in univariate analysis ( $p<0.001$ for each). Although immunosuppression was detected as a risk factor in multivariate analysis, 
the subgroups were not significantly found in multivariate analysis. Insufficiency of the number of cases may be the possible cause. In a study published by Wu in 2011, transplantation was found to be a risk factor in univariate analysis $(p=0,033)$ but surprisingly immunosuppressant use was not detected to be a risk factor itself so organ transplantation seems to be an RF in itself $[2,19]$. In our study, chemotherapy within the last 6 months was found to be statistically significant in univariate analysis. However, in patients who have received chemotherapy, it is not possible to only accuse chemotherapy sine there is underlying cancer. Furthermore, since chemotherapy is often given as a combination, further studies are needed in terms of detecting an isolated agent. Steroid was used at doses ranging from $1 \mathrm{~g} /$ day methylprednisolone to $4 \mathrm{mg} /$ day to dexamethasone, in the study. In subgroup analysis, once more excluding burns detected in multivariate analysis, all other factors were found to be more common in the steroid-using group. For this reason, although it is not possible to say for sure the effect steroid on which dose this effect starts or duration needed for this effect, it is already expected that the steroid will emerge as risk factor because it is known to suppress the cellular immune response.

Burn injuries are never sterile, despite the use of local and systemic antibiotics. The incidence of infections increases secondary to the loss of skin cover and the presence of conditions that develop after the trauma (eg acute renal failure, inhalation damage etc.) increase the risk of infection. Local defenses against infections are also deteriorated. In our study, when subgroup analysis was performed and evaluated in terms of other risk factors, burn seems to be an independent risk factor. Often these patients are younger, with no underlying chronic disease, although antibiotic use and central catheterization are more common than control group these numbers seems close to the case group. For this reason, although the literature has not been widely researched, the presence of burn seems to be an independent risk factor [20].

Central catheter and arterial catheter history is detected as a risk factor in multivariate analysis. In a study published in the United States in 2010, central venous catheters were reported as a risk factor for carbapenem-resistant Enterobacteraceae infection [19]. The presence of an arterial catheter is often accompanied by the presence of a central catheter. Patients with catheterization are frequently unable to receive oral, are septic, operated or in need of catheterization due to general condition. However, despite the fact that the presence of the catheter in these patients was at different rates, the presence of the catheter was statistically significant. However, independent of all these, catheterization has been reported in many publications as an isolated risk factor for gram-negative infections or colonization. In this case, the presence of a catheter seems to have to be accepted as an independent risk factor.

The presence of a feeding tube or nasogastric tube was detected as an independent risk factor in multivariate analysis. They are often used in patients where oral intake is not possible or oral intake is disadvantageous. Although it is safer and more useful in many respects than the parenteral route, in long term, inadequate nutrition, aspiration and bacterial translocation in the gastrointestinal system, especially in patients receiving vasopressor support, are frequent complications. In a study published by Falagas in Greece in 2007, nutrition tube presence was detected as a risk factor for carbapenem resistant Klebsiella pneumoniae [5]. The result of our work seems to be consistent with these results.

Elective surgical intervention was found to be statistically significantly different but not as a risk factor but as a protective factor. Subgroup analyzes of patients who underwent elective surgery showed fewer hospital stay duration, less underlying illness, less immunosuppression, younger age, less invasive intervention, and fewer antibiotics, as the probability of this condition was rather low. In addition, elective surgery was significantly higher in the control group than in the case group. Elective surgery may be interpreted as an indirect indication that the general condition of the patient is better rather than assuming it as a protective factor in our study.

Sulbactam/ampicillin, glycopeptide antibiotic and piperacillin/tazobactam or carbapenem use were detected as risk factors in multivariate analysis. It has been known for many years that the use of antibiotics increases the antibiotic resistance of bacteriae, and it is also known that risk increases parallel with the number of antibiotics used and the duration of antibiotic use. Sulbactam/ampicillin, glycopeptide antibiotics, piperacillin/tazobactam, and carbapenem use are all detected to increase the risk of carbapenem resistant E. coli, P. aeruginosa infection $[1,5,20,21]$. The result of our study seems to overlap with this. The reason for using carbapenem or piperacillin / tazobactam in patients in multivariate analysis is that in our hospital in cases where antipseudomonal, anaerobic and gram-negative efficacy is needed before carbapenem use piperacillin/ tazobaktam use is preferred unless the general condition of the patient is favorable. Subgroup analysis also showed that more than $80 \%$ of patients who used carbapenem had previously used piperacillin/ tazobactam. In fact, in multivariate analysis these 
two antibiotics were separately detected as risk factors.

\section{CONCLUSION}

In our hospital, carbapenem resistant K. pneumonia (71\%) infections/colonizations are more common than carbapenem resistant E. coli infections/colonizations (29\%).

The risk is more pronounced in internal wards, especially in NICU; but there is a lot of work to be done before it is accepted as an independent risk factor because of the many accompanying conditions of internal ward admission. There is insufficient data to say that internal ward need to be more careful in terms of infection control than surgical services. DM presence, previous hospitalization, burn history and immunosuppression were found among independent risk factors. Better blood glucose control in patients may lead to a reduction in risk for carbapenem resistant infections in DM patients, early isolation of burn patients may be beneficial for the patient and other patients in hospital, if infection develops in the presence of immunosuppression, carbapenem resistance should be kept in mind and should be evaluated in antibiotic therapy to be initiated. Steroid use should be in the presence of indication as much as possible.
[1] McConville TH, Sullivan SB, Gomez-Simmonds A, et al. Carbapenem-resistant Enterobacteriaceae colonization (CRE) and subsequent risk of infection and 90-day mortality in critically ill patients, an observational study. PLoS One 2017; 12(10): e0186195.

[2] Hong T, Moland ES, Abdalhamid B, et al. Escherichia coli: development of carbapenem resistance during therapy. Clin Infect Dis 2005; 40(10): e84-6.

[3] MacKenzie FM, Forbes KJ, Dorai-John T, et al. Emergence of a carbapenem-resistant Klebsiella pneumoniae. Lancet 1997; 350: 783 .

[4] Hussein K, Sprecher H, Mashiach T, et al. Carbapenem resistance among Klebsiella pneumoniae isolates: risk factors, molecular characteristics, and susceptibility patterns. Infect Control Hosp Epidemiol 2009; 30(7): 666-71.

[5] Falagas ME, Rafailidis PI, Kofteridis $D$, et al. Risk factors of carbapenem-resistant Klebsiella pneumoniae infections: a matched case control study. J Antimicrob Chemother 2007; 60(5): 1124-30

[6] Hoban DJ, Bouchillon SK, Hawser SP, et al. Trends in the frequency of multiple drug-resistant Enterobacteriaceae and their susceptibility to ertapenem, imipenem, and other antimicrobial agents: data from the Study for Monitoring Antimicrobial Resistance Trends 2002 to 2007. Diagn Microbiol Infect Dis 2010; 66(1): 78-86.

[7] Kwak YG, Choi SH, Choo EJ, et al. Risk factors for the acquisition of carbapenem-resistant Klebsiella pneumoniae among hospitalized patients. Microb Drug Resist 2005; 11(2): 165-9.

[8] Leavitt A, Navon-Venezia S, Chmelnitsky I, et al. Emergence of KPC-2 and KPC-3 in carbapenem-resistant Klebsiella pneumoniae strains in an Israeli hospital. Antimicrob Agents Chemother 2007; 51(8): 3026-9.

[9] Hossain A, Ferraro MJ, Pino M, et al. Plasmid-mediated carbapenem-hydrolyzing enzyme KPC-2 in an Enterobacter sp. Antimicrob Agents Chemother 2004; 48(11): 4438-40.

[10] Marra AR, Pereira CA, Gales AC, et al. Bloodstream infections with metallo-beta-lactamase-producing Pseudomonas aeruginosa: epidemiology, microbiology, and clinical outcomes. Antimicrob Agents Chemother 2006; 50(1): 388-90.

[11] Souli M, Kontopidou FV, Papadomichelakis E, et al.
Clinical experience of serious infections caused by Enterobacteriaceae producing VIM-1 metallo-beta-lactamase in a Greek University Hospital. Clin Infect Dis 2008; 46(6): 847-54.

[12] Hirakata H, Izumikawa K, Yamaguchi T, et al. Rapid detection and evaluation of clinical characteristics of emerging multiple-drug-resistant gram-negative rods carrying the metallo-beta-lactamase gene blalMP. Antimicrob Agents Chemother 1998; 42(8): 2006-11.

[13] Hancock RE. Resistance mechanisms in pseudomonas aeroginosa and other nonfermentative gram negative bacteria. Clin Infect Dis 1998; 27(1): 93-9.

[14] Duygu G. Gram negatif bakterilerde antibakteriyel direnç mekanizmaları, önemli ve sorunlu gram negatif bakteri infeksiyonları. 3. ed. İzmir; Palme Yayınevi, 2004; 69-83.

[15] Pitout JD, Gregson DB, Poirel L, et al. Detection of Pseudomonas aeruginosa producing metallo-beta-lactamases in a large centralized laboratory. J Clin Microbiol 2005; 43(7): 3129-35.

[16] Turton JF, Ward ME, Woodford N, et al. The role of ISAba1 in expression of OXA carbapenemase genes in Acinetobacter baumannii. FEMS Microbiol Lett 2006; 258(1): 72-7.

[17] Potter RF, D'SouzaA, Dantas G. The rapid spread of carbapenem-resistant Enterobacteriaceae. Drug Resist Updat 2016; 29: 30-46.

[18] Guidance for Control of Infections with CarbapenemResistant or Carbapenemase-Producing Enterobacteriaceae in Acute Care Facilitiess. CDC 2009; 58 (10): 256-260.

[19] Wu UI, Wang JL, Chen WC, et al. Risk factors and outcomes of Escherichia coli bacteremia caused by strains that produce CTX-M or non-CTX-M extended-spectrum-beta-lactamases. Eur J Clin Microbiol Infect Dis 2011; 30(1): 33-9.

[20] Salomão MC, Duailibi DF, Perondi MBM, et al. Carbapenemresistant Enterobacteriaceae in patients admitted to the emergency department: prevalence, risk factors, and acquisition rate. J Hospt Infect 2017; 97(3): 241-246.

[21] Hussein K, Sprecher H, Mashiach T, et I. Carbapenem resistance among Klebsiella pneumonia Isolates: Risk Factors, Molecular Characteristics, and Susceptibility Patterns. Infect Control Hosp Epidemiol 2009; 30(7): 666-71. 